\title{
Evidence of intermittency in the local field potentials recorded from patients with Parkinson's disease: a wavelet-based approach
}

\begin{abstract}
ASOK K. SEN†* and JONATHAN O. DOSTROVSKY
$\dagger$ Department of Mathematical Sciences, Indiana University, 402 N. Blackford Street, Indianapolis, IN 46202, USA \$Department of Physiology, University of Toronto, 1 King’s College Circle, Toronto, ON M5S 1A8, USA

(Received 15 January 2007; revised 24 May 2007; in final form 8 June 2007)

Using a continuous wavelet transform we have detected the presence of intermittency in the beta oscillations of the local field potentials (LFPs) that were recorded from the subthalamic nucleus (STN) of patients with Parkinson's disease. The intermittent behavior was identified by plotting the wavelet power spectrum of the LFP signal on a time-frequency plane. We also computed the temporal variations of scale-averaged wavelet power and wavelet entropy (WE). An intermittent pattern is characterized by large amounts of power over very short periods of time separated by almost quiescent periods. Time-localized changes in WE further support the evidence of intermittency. The cause and significance of the intermittent beta activity are presently unclear. It may be due to complex interactions of the cortico-basalganglia networks converging at the STN level.
\end{abstract}

Keywords: Local field potentials; Parkinson's disease; Subthalamic nucleus; Basal ganglia

\section{Introduction}

The basal ganglia (BG) have been the subject of intense investigation because of their relevance in the pathophysiology of movement disorders such as Parkinson's disease (PD). In the past few years there has been increasing interest in studying the possible role of oscillatory activity in the BG [1,2], and in particular, in the subthalamic nucleus (STN), a key nucleus of the BG. High-frequency stimulation through deep brain stimulation (DBS) electrodes implanted in the STN has been found to greatly alleviate the motor symptoms of PD patients although the mechanism of DBS is still poorly understood [3]. Spectral analysis of local field potentials (LFPs) recorded from the contacts of DBS electrodes has revealed that in PD patients off dopaminergic medication there is prominent power in the $13-30 \mathrm{~Hz}$ band, known as the beta band [3-5]. Interestingly the beta power greatly decreases with dopaminergic medication [4-8], and it has been proposed that the Parkinsonian symptoms may be due to increased beta oscillatory activity in STN and that the DBS induced reduction in symptoms may be due to disruption of this oscillatory activity [9].

The use of microelectrode recordings to help localize the optimum site for placement of DBS electrodes provides a unique opportunity to obtain data on neuronal firing rates and

\footnotetext{
*Corresponding author. Email: asen@inpui.edu
} 
LFPs within the STN during surgery. Recent microelectrode recordings in the STN of PD patients has revealed the existence of neurons whose firing is oscillatory and coherent with beta oscillatory LFPs [5,7]. It has also been postulated that these oscillations may arise from synchronized neuronal discharge in the cortex [10]. There is currently a great deal of interest in understanding the properties and possible function of this beta band of oscillations in Parkinsonian patients. The aim of this study is to demonstrate the presence of intermittency in the beta oscillations. An intermittent pattern is characterized by large amounts of power over short periods of time separated by almost quiescent periods. Our approach is based on wavelet analysis which is a practical method for time-dependent spectral analysis of experimental data.

Wavelets have been used for the analysis of time series in a wide variety of applications including biomedical signal processing [11-14]. They are particularly suited for spectral analysis of nonstationary signals. A wavelet-based approach has advantages over the more traditional methods such as the Fourier transform or the windowed Fourier transform. The Fourier transform is a purely frequency domain technique which seeks to determine the periodicities of a signal through spectral peaks; it cannot, however, determine the temporal variations of these periodicities, if any. A simple way to capture the temporal variations is to apply Fourier transform on a short segment of the signal at a time by windowing the signal and then sliding the window in time. This procedure is known as the Short-Time Fourier Transform (STFT) or windowed Fourier transform. But, due to the fact that STFT uses a fixed-size window, it suffers from the following drawback. For a given signal, its frequency resolution may be poor or its temporal resolution may be less precise, depending on the chosen window size. Using a variable-size window in a time-frequency plane, wavelet analysis provides an efficient approach by which both time and frequency resolutions can be adjusted in an adaptive fashion. A wavelet transform uses a window that narrows when focusing on small-scale or high-frequency features of the signal and widens on large-scale or low-frequency features, analogous to a zoom lens [13].

Wavelet analysis has also been found to be useful for detecting intermittent behavior, particularly in the area of fluid mechanics (see, for example, [15-17]). Among other applications, Rybak et al. [18] noticed an intermittent pattern in solar flare activity with the aid of a continuous wavelet transform (CWT). In addition, the concept of wavelet entropy (WE) as a measure of complexity has been used in many applications [19-22]. In this paper we use a CWT to analyze the oscillatory activity of the LFPs in STN and demonstrate the presence of intermittency in the beta frequency band.

\section{Data acquisition}

The LFPs were recorded from the STN of patients with PD who were undergoing stereotactic surgery for implantation of DBS electrodes. The studies were approved by the University Health Network Ethical Review Board and University of Toronto and patients gave written and informed consent prior to surgery. Details of the recording procedure employed have been described in our previous publications (see, for example, [6]). Briefly, LFPs were recorded from a microelectrode as it was driven through the STN under local anesthesia. Recordings were amplified, band-pass filtered and digitized at $1 \mathrm{kHz}$. Records were obtained when the patients were at rest. Patients had been off dopaminergic medication for over $12 \mathrm{~h}$ at the time of the recordings. We analyzed the recordings from several PD patients and also from different locations within the STN of each patient. 


\section{Wavelet analysis of intermittency}

First we calculated the wavelet power spectrum (WPS) of the LFP signal using a CWT and plotted the WPS on a time-frequency plane as a contour plot. The intermittent pattern of the beta oscillations can be clearly identified from the time-frequency landscape by visual inspection. We also computed the scale-averaged wavelet power and WE and plotted their variations with time. Sudden bursts of power with intervening periods of significantly reduced power reveal the characteristic intermittent pattern. Time-localized changes in WE further support the evidence of intermittent beta activity.

A wavelet is a small wave that is localized in time. In order to be classified as a wavelet, a function $\psi(t)$ should have zero mean and finite energy:

$$
\int_{-\infty}^{\infty} \psi(t) \mathrm{d} t=0, \quad \int_{-\infty}^{\infty}|\psi(t)|^{2} \mathrm{~d} t<\infty .
$$

The CWT of a signal $x(t)$ with respect to a wavelet $\psi(t)$ is defined as a convolution of the signal with a scaled and translated version of $\psi(t)$. The wavelet $\psi(t)$ is referred to as an analyzing wavelet or a mother wavelet and $t$ denotes time. The convolution is expressed by the integral [13]:

$$
W(s, t)=\int_{-\infty}^{\infty} x\left(t^{\prime}\right) \psi_{s, t}^{*}\left(t^{\prime}\right) \mathrm{d} t^{\prime}
$$

where

$$
\psi_{s, t}\left(t^{\prime}\right)=\frac{1}{\sqrt{s}} \psi\left(\frac{t^{\prime}-t}{s}\right)
$$

is a scaled and translated version of the mother wavelet $\psi(t)$, and an asterisk on $\psi$ denotes its complex conjugate. The symbol $s$ represents the scale parameter and $t$ is time. The scale parameter controls the dilation $(s>1)$ and contraction $(s<1)$ of the wavelet. The factor $1 / \sqrt{s}$ is introduced in equation (3) so that $\psi_{s, t}\left(t^{\prime}\right)$ has unit energy at every scale. The WPS of the signal representing the energy at scale $s$ is defined as the squared modulus of the CWT:

$$
P(s, t)=|W(s, t)|^{2} .
$$

Using this WPS, a time-dependent WE for the signal can be formulated as follows.

$$
W E=-\sum_{s} p_{s, t} \log _{2} p_{s, t}
$$

with

$$
p_{s, t}=\frac{P(s, t)}{\int_{0}^{\infty} P(s, t)(\mathrm{d} s) /\left(s^{2}\right)}
$$

representing the probability distribution of the signal energy at each scale. WE can be interpreted as a measure of signal complexity [19]. 
The integral formulation of a CWT as given in equation (1) applies to a signal $x(t)$ that is a continuous function of time. In order to use it for a discrete signal such as the LFP signal, this integral representation must be discretized in an appropriate fashion. Consider a time series $\left\{x_{n}\right\}, i=1,2,3, \ldots, N$. For this time series, equation (1) may be discretized as [23]:

$$
W_{n}(s)=\sum_{n^{\prime}=1}^{N}\left(\frac{\delta t}{s}\right)^{1 / 2} x_{n^{\prime}} \psi^{*}\left[\frac{\left(n^{\prime}-n\right) \delta t}{s}\right] .
$$

Here $n$ is the time index, and $\delta t$ is the sampling interval. It has been shown by Torrence and Compo [23] that using a discrete Fourier transform, all $N$ convolutions in equation (7) can be performed simultaneously in Fourier space and thus the wavelet transform $W_{n}(s)$ can be calculated in an efficient manner.

Using equation (7), the WPS of a discrete signal can be evaluated as: $\left|W_{n}(s)\right|^{2}$. This power spectrum which depends on both scale and time is represented by a surface. By taking contours of this surface and plotting them on a plane, a time-scale representation of the spectrum may be derived. A time-scale representation is found to be useful for extracting important features of signals arising in several applications such as climate dynamics [13]. An alternate representation, namely, a time-frequency representation has also been used for the purpose of feature extraction in many applications. A scale-to-frequency conversion, which follows a reciprocal relationship, can be easily made by use of the formula: $f=f_{0} f_{*} / s$, where $f$ is the instantaneous frequency of the signal, $f_{*}$ is the sampling frequency, and $f_{0}$ is the center frequency of the mother wavelet (see below). In our analysis we used a complex Morlet wavelet as the mother wavelet. Morlet wavelets have been used earlier for the analysis of neurophysiological signals [24,25]. A complex Morlet wavelet consists of a complex sinusoid modulated by a Gaussian function and is described by:

$$
\psi(\eta)=\pi^{-1 / 4} \mathrm{e}^{i \omega_{0} \eta} \mathrm{e}^{-\eta^{2} / 2}
$$

Here $\omega_{0}=2 \pi f_{0}$, with $f_{0}$ being the center frequency. The angular frequency $\omega_{0}$ is often referred to as the order of the wavelet. It should be noted that the mother wavelet as defined in equation (8) is strictly not a wavelet since it does not have zero mean. The zero mean property can be satisfied by multiplying the right-hand side of equation (8) by a correction factor. However, if $\omega_{0}>5$, this correction factor is negligible and equation (8) can be used without loss of accuracy. In our computations we have used $\omega_{0}=6$. This choice for $\omega_{0}$ offers a good balance between time and frequency localizations.

\section{Results and discussion}

Figure 1 depicts a representative time series of the LFP signal recorded from the STN of a PD patient. The time series consists of an 85 -second recording and is obtained by sampling the LFP signal at $1000 \mathrm{~Hz}$.

Figure 2 shows a time-frequency representation of the WPS of the LFP signal presented in figure 1. As indicated above, this is obtained with a Morlet mother wavelet of order 6 . The color map in this figure ranges from blue to red according to low and high power levels, respectively. From the computations of the WPS we find that the beta oscillations have maximum power at approximately $14 \mathrm{~Hz}$; this is also seen from the time-frequency plot shown in figure 2. There is also significant power between 8 and $20 \mathrm{~Hz}$. It is apparent 


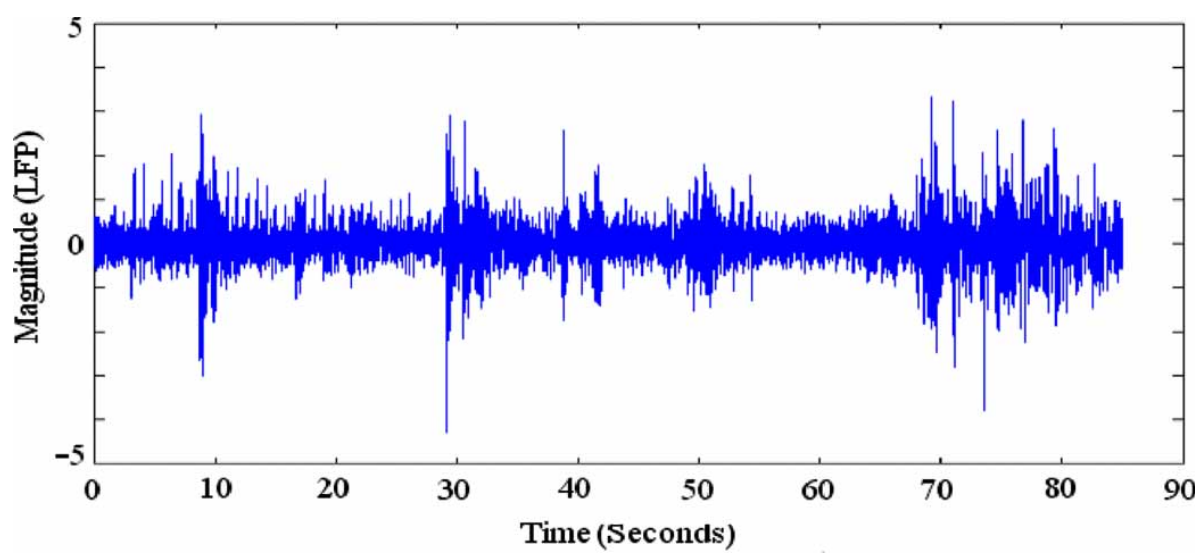

Figure 1. A time series of the LFP signal sampled at $1000 \mathrm{~Hz}$. The magnitudes of the LFP are in arbitrary units.

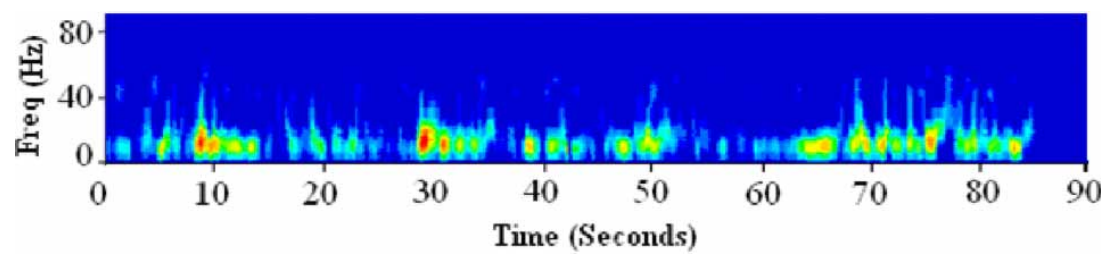

Figure 2. A time-frequency representation of the WPS of the LFP signal shown in figure 1. This is obtained using a CWT with a Morlet wavelet of order 12 as the mother wavelet.

in figure 2 that there are bursts of power of beta activity over very short intervals of time with periods of significantly reduced beta activity or almost no beta activity in between, revealing the presence of intermittency. To our knowledge, this intermittent behavior of the LFP beta activity in the STN of PD patients under resting conditions has not been reported previously.

We have also calculated the scale-averaged wavelet power across time for the LFP signal shown in figure 1 . This is done by integrating the WPS over the frequency range of $8-20 \mathrm{~Hz}$ (figure 2). The variation of the wavelet power with time is displayed in figure 3 . The irregular bursts of power separated by almost quiescent periods are evident in this figure, indicating an intermittent behavior. In addition, we have computed the WE of the LFP signal using equation (5). The WE is plotted as a function of time in figure 4. The time-localized changes in WE support the evidence of intermittency in the beta oscillations.

A comparison of figures 3 and 4 shows that there is a correlation between the transient dominant energy peaks and a decrease in time-localized WE. This negative correlation is supported by the fact that a decrease in entropy typically characterizes synchronized neuronal interactions [26]. In particular, the time at which the WE is at a minimum can be associated with highly synchronized neuronal activity in the beta band. An increase in entropy, on the other hand, may correspond to blocking of neuronal activity [21].

Although there have been many studies over the past 5 years documenting beta oscillatory activity in the STN of PD patients, the intermittent nature of this activity has not been reported earlier. Previous studies have, however, shown that the beta activity is decreased by dopaminergic medication and movements and the changes can be time-locked to the onset of the movement $[4-8,27]$. The intermittency reported here appears to be a very general characteristic of the beta oscillatory activity in the STN since it was observed in all patients 


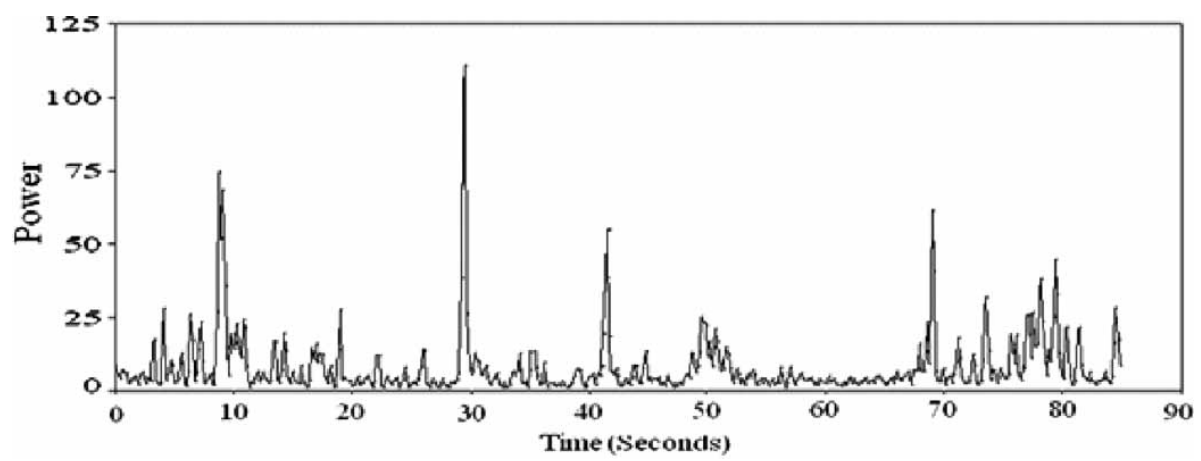

Figure 3. Variation of scale-averaged wavelet power with time for the LFP signal shown in figure 1.

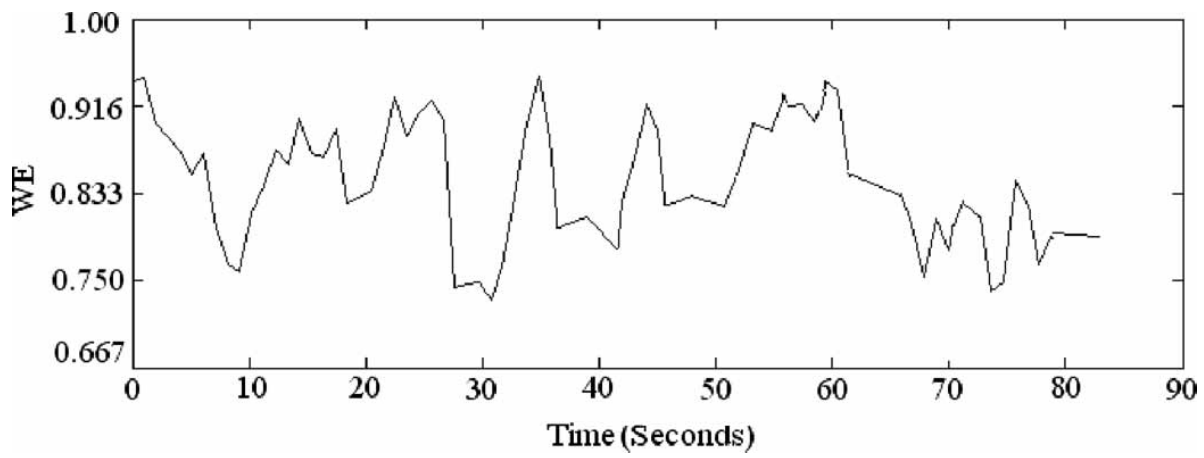

Figure 4. Variation of WE with time for the LFP signal shown in figure 1. Normalized values of the entropy are plotted here.

and most sites we have examined. Since the origin of the beta oscillations has not been established, it is difficult to speculate on why it is intermittent. It may be due to complex interactions of the cortico-basal-ganglia networks which converge at the STN level.

Using the wavelet-based techniques employed in this paper, it will be useful to examine how movement can influence the beta activity in the presence of intermittency. Such studies may provide clues to the relevance of intermittent behavior to the normal and/or pathophysiological function of the STN.

\section{Acknowledgements}

We would like to thank Moran Weinberger for her help with this project, and Drs W. D. Hutchison and A. M. Lozano for their help in obtaining the recordings. This work was supported by a grant from the Canadian Institutes of Health Research (CIHR-MOP-42505).

\section{References}

[1] Brown, P. and Williams, D., 2005, Basal ganglia local field potential activity: character and functional significance in the human, Clinical Neurophysiology, 116, 2510-2519. 
[2] Hutchison, W.D., Dostrovsky, J.O., Walters, J.R., Courtemanche, R., Boraud, T., Goldberg, J. and Brown, P., 2004, Neuronal oscillations in the basal ganglia and movement disorders: evidence from whole animals and human recordings, The Journal of Neuroscience, 24, 9240-9243.

[3] Lozano, A.M., Dostrovsky, J.O., Chen, R. and Ashby, P., 2002, Deep brain stimulation for Parkinson's disease: disrupting the disruption, Lancet, 1, 225-231.

[4] Cassidy, M., Mazzone, P., Oliviero, A., Insola, A., Tonali, P., Di Lazzaro, V. and Brown, P., 2002, Movement related changes in synchronization in the human basal ganglia, Brain, 125, 1235-1246.

[5] Kuhn, A.A., Trottenberg, T., Kivi, A., Kupsch, A., Schneider, G-H. and Brown, P., 2005, The relationship between local field potential and neuronal discharge in the subthalamic nucleus of patients with Parkinson's disease, Clinical Neurophysiology, 194, 212-220.

[6] Levy, R., Hutchison, W.D., Lozano, A.M. and Dostrovsky, J.O., 2002, Synchronous neuronal discharge in the basal ganglia of Parkinson's patients is limited to oscillatory activity, The Journal of Neuroscience, 22, 2855-2861.

[7] Doyle, L.M., Kuhn, A.A., Hariz, M., Kupsch, A., Schneider, G.H. and Brown, P., 2005, Levadopa-induced modulation of subthalamic beta oscillations during self-paced movements in patients with Parkinson's disease, The European Journal of Neuroscience, 23, 1956-1960.

[8] Williams, D., Tijssen, M., van Bruggen, G., Bosch, A., Insola, A., Di Lazzaro, V., Mazzone, P., Oliviero, A., Quartarone, A., Speelman, H. and Brown, P., 2003, Dopamine-dependent changes in the functional connectivity between basal ganglia and cerebral cortex in humans, Brain, 125, 1558-1569.

[9] Wingeier, B., Tcheng, T., Koop, M.M., Hill, B.C., Heit, G. and Bonte-Stewart, H.M., 2006, Intra-operative STN DBS attenuates the prominent beta activity in the STN in Parkinson's dsease, Experimental Neurology, 197, 244-251.

[10] Magill, P.J., Sharott, A., Bevan, M.D., Brown, P. and Bolam, J.P., 2004, Synchronous unit activity and local field potentials evoked in the subthalamic nucleus by cortical stimulation, Journal of Neurophysiology, 92, 700-714.

[11] Akay, M., 1997, Time-Frequency and Wavelets in Biomedical Signal Processing (New York: John Wiley \& Sons).

[12] Gencay, R., Selcuk, F. and Whitcher, B., 2001, An Introduction to Wavelets and Other Filtering Methods in Finance and Economics (London: Academic Press).

[13] Kumar, P. and Foufoula-Georgiou, E., 1997, Wavelet analysis for geophysical applications, Reviews of Geophysics, 35, 385-412.

[14] Sen, A.K., Litak, G., Taccani, R. and Radu, R., 2007, Wavelet analysis of cycle-to-cycle pressure variations in an internal combustion engine, Chaos Solitons and Fractals (in press).

[15] Chainais, P., Abry, P. and Pinton, J.-F., 1999, Intermittency and coherent structures in a swirling flow: a wavelet analysis of pressure and velocity measurements, Physics of Fluids, 11, 3524-3539.

[16] Nicolleau, F. and Vassilicos, J.C., 1999, Wavelets for the study of intermittency and its topology, Philosophical Transactions of the Royal Society of London Series A, 357, 2439-2457.

[17] Addison, P.S., Murray, K.B. and Watson, J.N., 2001, Wavelet transform analysis of open channel wake flows, ASCE Journal of Engineering Mechanics, 127, 58-70.

[18] Rybak, J., Ozguc, A., Atac, T. and Sozen, E., 2005, Intermittence of short-term periodicities of the flare index, Advances in the Space Research, 35, 406-409.

[19] Sello, S., 2000, Wavelet entropy as a measure of solar cycle complexity, Astronomy and Astrophysics, 363, 311-315.

[20] Quian Quiroga, R., Rosso, O.A. and Basar, E., 1999, Wavelet entropy: a measure of order in evoked potentials, Electroencephalography and Clinical Neurophysiology Supplement, 49, 299-303.

[21] Rosso, O.A., Blanco, S., Yordanova, J., Kolev, V., Figliola, A., Schurmann, M. and Basar, E., 2003, Wavelet entropy: a new tool for analysis of short duration brain electrical signals, Journal of Neuroscience Methods, 105, 65-75.

[22] Rosso, O.A., Blanco, S. and Rabinowicz, A., 2003, Wavelet analysis of generalized tonic-clonic seizures, Signal Processing, 83, 1275-1289.

[23] Torrence, C. and Compo, G.P., 1998, A practical guide to wavelet analysis, Bulletin of the American Meteorological Society, 79, 61-78.

[24] Andino, S.L.G., Menendez, R.G.P., Thut, G., Spinelli, L., Blanke, O., Michel, C.M., Seeck, M. and Landis, T., 2000, Measuring the complexity of time series: an application to neurophysiological signals, Human Brain Mapping, 11, 46-57.

[25] Samar, V.J., Bopardikar, A., Rao, R. and Swartz, K., 1999, Wavelet analysis of neuroelectric waveforms: a conceptual tutorial, Brain and Language, 66, 7-60.

[26] Lehnertz, K. and Elger, C.E., 1995, Spatio-temporal dynamics of the primary epileptogenic area in temporal lobe epilepsy characterized by neuronal complexity loss, Electroencephalography and Clinical Neurophysiology, 95, 108-117.

[27] Kuhn, A.A., Williams, D., Kupsch, A., Limousin, P., Hariz, M., Schneider, G.H. and Brown, P., 2004, Event-related beta desynchronization in human subthalamic nucleus correlates with motor performance, Brain, 127, 735-746. 


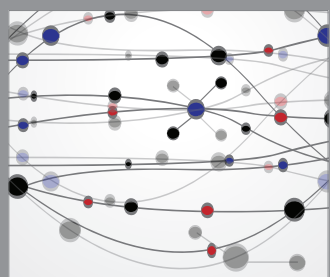

The Scientific World Journal
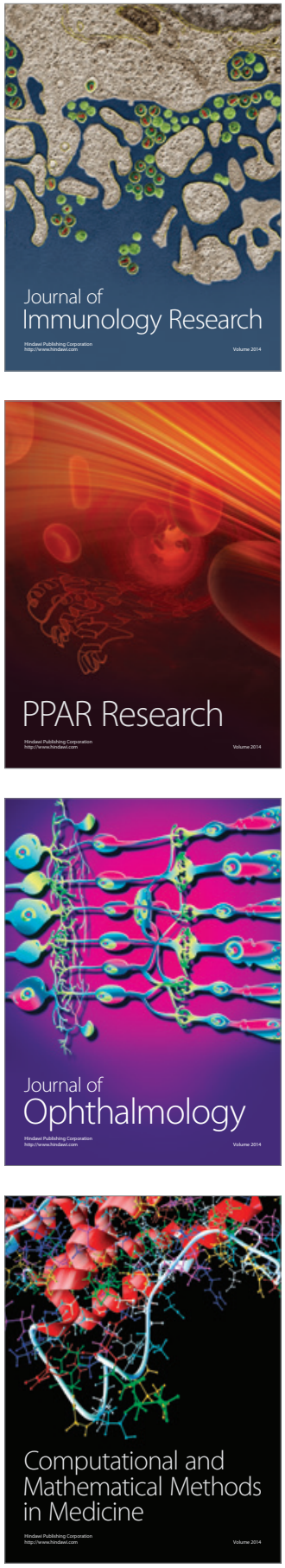

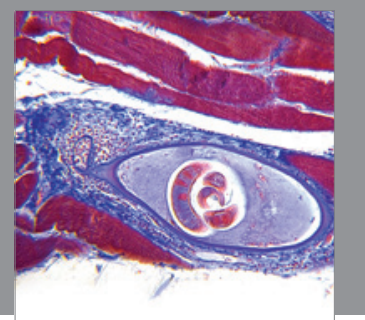

Gastroenterology

Research and Practice
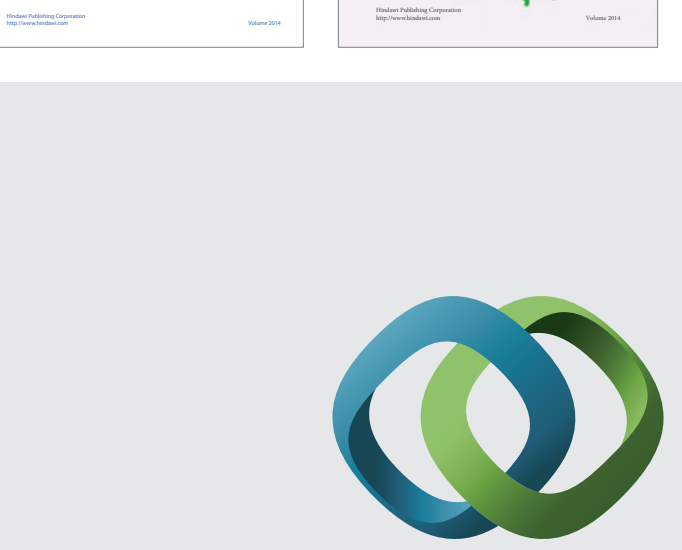

\section{Hindawi}

Submit your manuscripts at

http://www.hindawi.com
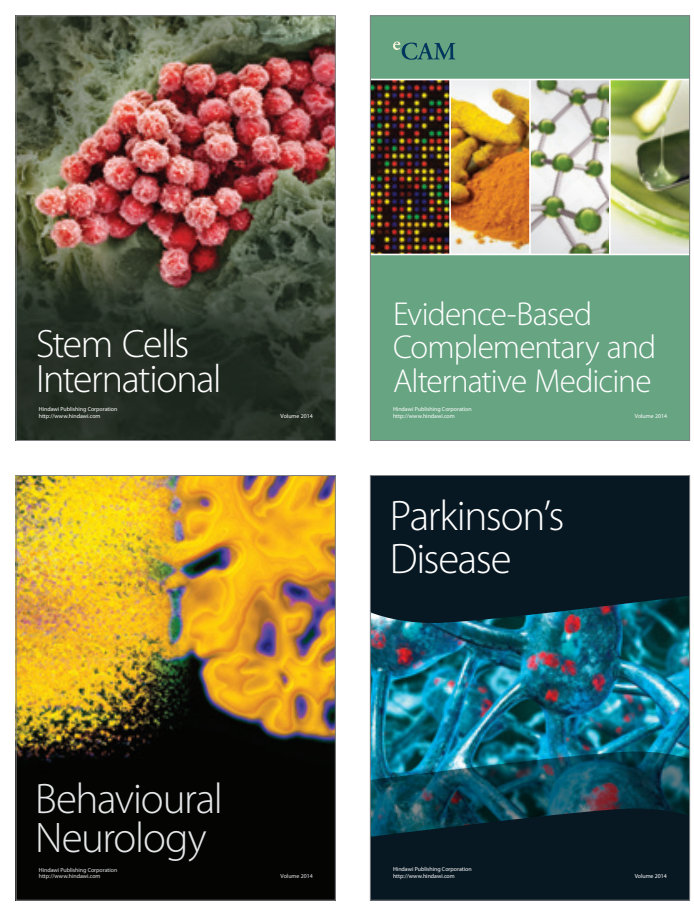

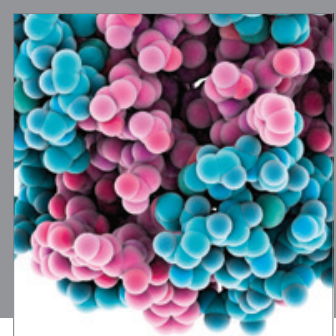

Journal of
Diabetes Research

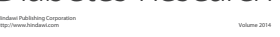

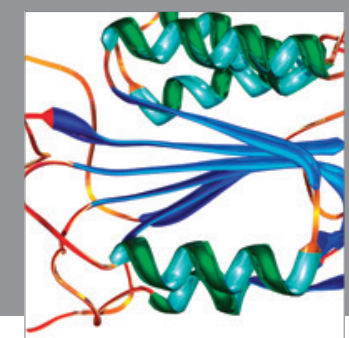

Disease Markers
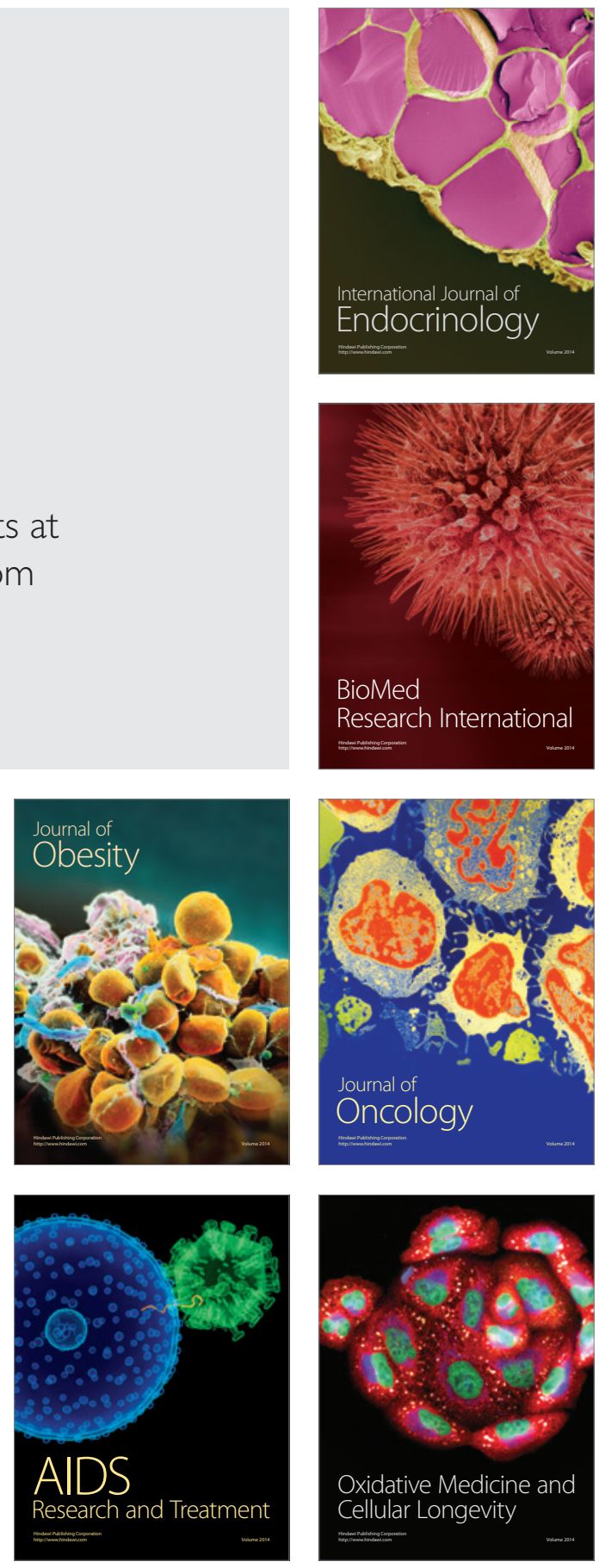